\title{
Review Article \\ Relevance of VEGF and Nephrin Expression in Glomerular Diseases
}

\author{
Claudia A. Bertuccio \\ Department of Cell Biology and Physiology, University of Pittsburgh, Pittsburgh, PA 15261, USA \\ Correspondence should be addressed to Claudia A. Bertuccio, cab199@pitt.edu \\ Received 16 March 2011; Accepted 25 May 2011 \\ Academic Editor: Mira Krendel
}

Copyright ( $) 2011$ Claudia A. Bertuccio. This is an open access article distributed under the Creative Commons Attribution License, which permits unrestricted use, distribution, and reproduction in any medium, provided the original work is properly cited.

The glomerular filtration barrier is affected in a large number of acquired and inherited diseases resulting in extensive leakage of plasma albumin and larger proteins, leading to nephrotic syndrome and end-stage renal disease. Unfortunately, the molecular mechanisms governing the development of the nephrotic syndrome remain poorly understood. Here, I give an overview of recent investigations that have focused on characterizing the interrelationships between the slit diaphragm components and podocytessecreted VEGF, which have a significant role for maintaining the normal podocyte structure and the integrity of the filtering barrier.

\section{Characteristic of the Glomerular Filtration Barrier and the Slit Diaphragm}

The cells of the human body are surrounded by liquid that is remarkably constant in its contents. The continued regulation of the dissolved compounds and ions in this internal environment is referred as homeostasis. Our kidneys are extremely important in preserving the homeostasis. They work rapidly and sensitively in order to regulate the excretion of unwanted and toxic products resulting from tissue metabolism, hence maintaining optimum concentration of the fluids, electrolytes, and acid-base in the body. The kidney is formed by an estimated number of renal glomeruli of $\sim 1$ million that filtrate approximately 180 liters of plasma every day, and only about 1.5-1.8 liters of this filtrate are excreted as urine. The plasma molecules are sieved through the renal glomerular filtration barrier based on their size and charge [1]. This barrier is a highly specialized structure which consists of three layers: a fenestrated endothelium, a 300-350 nm thick glomerular basement membrane (GBM), and the podocytes. The luminal side of the fenestrated endothelial cells is covered by a layer of negatively charged membranebound macromolecules (proteoglycans, glycosaminoglycans, glycoproteins, and glycolipids) named glycocalyx, and it is important for the selectivity properties of the glomerulus [2].
The GBM is composed of glycoproteins including type IV collagen, laminins, entactin/nidogen, and heparin sulphate proteoglycans such as agrin and perlecan. Type IV collagen and laminins form complexes which are connected by entactin/nidogen. This association plays an essential role in size selectively restricting the passage of the proteins through the glomerular filter [3-5]. The podocytes are highly differentiated cells, and they are the visceral epithelial cells of the kidney glomerulus. The podocytes project primary processes from the cell body, which further branch into numerous finger like processes called foot processes that enwrap the glomerular capillaries. The neighboring foot processes derived from different cell bodies are connected by a continuous adherent junction structure called the slit diaphragm (SD) [6]. The apical membrane above the SD is highly negatively charged mainly due to a heavily sialylated glycoprotein podocalyxin [7]. The basal membrane of podocyte foot processes serves to anchor podocytes to the GBM via several adhesion molecules including $\alpha 3 \beta 1$ integrin complex [8] and $\alpha$ - and $\beta$-dystroglycans [9]. On the other hand, the structure of the podocyte is maintained by a complex cytoskeleton consisting of actin, myosin, $\alpha$-actinin4 , talin, and vinculin [10]. When podocytes are injured, podocytes undergo a dramatic morphological change named foot process effacement resulting from alteration in its 
cytoskeletal structure and intercellular junctions. A direct correlation between this process and the development of proteinuria both in human disease and in experimental models has been shown [11].

The slit diaphragm expresses several members of the immunoglobulin (Ig) and cadherin superfamilies. Nephrin, a member of the Ig superfamily, is a transmembrane protein of $180 \mathrm{kDa}$ in humans, and it is posttranslationally modified by $\mathrm{N}$-glycosylation [12]. It has an extracellular segment containing $8 \mathrm{Ig}$-like domains, a fibronectin domain and the 10 putative $\mathrm{N}$-glycosylation sites, a transmembrane region, and a short cytoplasmic segment [12-14]. It has been shown that the N-glycosylation of nephrin is important for its appropriate folding and localization in the plasma membrane [15]. In 1998, it was suggested that nephrin is the structural backbone of the slit diaphragm [12]. Several studies have provided evidence that nephrin associates with itself [16]. Nephrin has three "free" cysteines in the extracellular domain, one in the Ig motif 1 , one in the spacer region between Ig motifs 6 and 7, and one in the fibronectin domain. These free cysteines residues in the extracellular domain allow the alignment between nephrin molecules from the neighboring foot processes in an antiparallel mode and bridge the distance between interdigitating podocyte foot processes $[14,17]$. The cytoplasmic region of nephrin contains nine conserved tyrosines residues, which could become phosphorylated during the binding of nephrin ligand and can serve as binding sites for the $\mathrm{SH} 2$ domains of cytoplasmic targets [18-21]. The natural extracellular ligands for nephrin remain unknown until now. In addition to the glomerular podocytes of kidneys, nephrin is expressed in the pancreas and in the nervous system [22]. Humans and rodents also express a splice variant of nephrin that excludes the transmembrane region [23]. Mutations of the nephrin gene, NPHS1, produce congenital nephrotic syndrome of the Finnish type (CNF) [12]. CNF is an autosomal, recessive disorder, characterized by massive proteinuria in utero, and the classical signs of nephrotic syndrome hypoalbuminemia, hyperlipidemia, and edema developed within days after birth. Kidney biopsy of children with CNF shows podocyte foot process effacement and absence of the slit diaphragms [24]. The disease usually leads to death in the neonatal period but can be treated with dialysis followed by kidney transplantation in early childhood [25].

A growing number of proteins, including Neph1, the classical P-cadherin that belongs to the Ig superfamily [6], FAT-1 a nonclassical cadherin superfamily member [26], and the tight junction protein ZO-1 [27] are localized at the SD. In the SD, the cytoplasmic tail of nephrin interacts with the adapter protein CD2-AP [28, 29], podocin [30,31], and the actin cytoskeleton via CD2-AP [32]. The C-terminal domain of podocin interacts with nephrin, Neph1 [33], and CD2-AP [30]. Neph1 also interacts with podocin [33] and ZO-1 [34]. The canonical transient receptor potential 6 ion channel (TRPC6) interacts with podocin and nephrin in the slit diaphragm [35]. Subsequently, it was shown that mutations in several of these slit diaphragm-associated proteins, including Neph1 [36], podocin [37], CD2-AP [29], FAT-1 [38], and TRPC6 [39] results in effacement of podocytes, heavy proteinuria, development of nephrotic syndrome, and early postnatal death in the majority on the cases. In addition, mutations in $\alpha$-actinin-4 [40], $\alpha 3 \beta 1$ integrin [8], and podocalyxin [41] can lead to the same phenotype. Taking together all these studies, it has been proposed that the slit diaphragm plays a major role in maintaining the filtration barrier of the renal glomerulus. However, the molecular components and function of the SD remain incompletely characterized.

\section{Altered VEGF-A and Slit Diaphragm Protein Levels Can Cause Proteinuria}

Several lines of evidence showed that vascular endothelial growth factor A (VEGF-A), the best characterized angiogenic/vasculogenic factor, is a critical "cross-talk" protein among the three components of the glomerular filtration barrier [42]. Patients who underwent chemotherapy with anti-VEGF (e.g., bevacizumab, sunitinib) or receptor tyrosine kinase inhibitors lead to proteinuria, podocyturia, hypertension, edema, glomerular capillary endotheliosis, and glomerular thrombotic microangiopathy indicating damage to the glomerular filtration barrier [43-46]. In addition, several reports showed that women with preeclampsia, a condition characterized by excess circulating levels of a soluble form of the VEGF receptor (sFlt-1, see below), developed similar symptoms that are in VEGF ablation therapy $[47,48]$. Experiments with normal healthy mice showed that intravenous infusion of sFlt-1 or anti-VEGF antibodies causes glomerular endotheliosis and proteinuria [49]. Taken together, these results emphasize the importance of the regulation of VEGF-A signaling in the development of proteinuria.

VEGF-A belongs to a family of multipotent cytokines. Six different VEGF-A isoforms and two known families can be generated by alternative splicing of eight exons separated by 7 introns of a single gene. The VEGFxxx family of isoforms, where $\mathrm{xxx}$ refers to the number of amino acids, is formed by differential splicing in exons 6 and 7 and the proximal splice site in exon 8 (termed exon $8 \mathrm{a}$ ). They are named VEGF 121, 145, 165, 183, 189, and 206 based on the number of amino acids in humans. Rodents present the same number of isoforms, although they are one amino acid shorter [50]. $\mathrm{VEGF}_{121}, \mathrm{VEGF}_{165}$, and $\mathrm{VEGF}_{189}$ are the most abundantly expressed isoforms, whereas $\mathrm{VEGF}_{145}$ and $\mathrm{VEGF}_{206}$ are comparatively rare [50]. In human kidneys, the most abundant isoforms of VEGF-A are strongly expressed in podocytes and also in proximal and distal tubular cells [51]. Receptors for VEGF-A are vascular endothelial growth factor receptor VEGFR-1 (also known as Flt-1) and VEGFR-2 (also known as Flk-1), which are predominantly expressed on the glomerular endothelial cells and pre- and postglomerular vessels [51, 52]. These VEGF receptors belong to the PDGF (platelet-derived growth factor) family of receptor tyrosine kinase (RTKs, class III) and have a similar domain structure [53]. VEGFR-1 and -2 contain three functional domains: seven Ig-like extracellular domains, a single membrane spanning region, and a cytoplasmic tyrosine kinase domain 
$[54,55]$. Binding of growth factors to the second and third immunoglobulin domains leads the receptors dimerization, protein kinase activation, autophosphorylation, and initiation of signaling pathways [56]. In addition to these receptors tyrosine kinases, VEGFxxx interacts with a family of coreceptors, the neuropilins (Np) [57]. Soluble truncated forms of both VEGFR-1 and VEGFR-2 have recently been found in plasma. Even though little is known about the latter [58], the soluble form of the VEGF receptor-1 (sFlt1) is known to regulate VEGF activity by binding VEGF-A in the circulation [59]. Park et al. proposed that full length VEGFR-1 and sFlt-1 have a "decoy" function on the vascular endothelium, where they exert a negative effect on VEGF activity, by preventing VEGF binding to VEGFR-2 [60].

Recently, another novel set of VEGF-A isoforms called "VEGFxxxb" have been described. The VEGFxxxb family is formed by splice variant using a distal site on exon 8 (termed exon 8b) [61, 62]. The VEGFxxxb transcripts code for polypeptides with the same length as the classical ones, because exon $8 \mathrm{a}$ and exon $8 \mathrm{~b}$ have the same size. Therefore, these isoforms were named VEGF121b, 145b, 165b, 183b, $189 \mathrm{~b}$, and 206b. Furthermore, it has been identified that VEGFxxxb interacts with the VEGFR-2 extracellular domain, resulting in differential downstream kinase activation, but the "b-isoforms" are unable to bind Np [63]. VEGF165b was the first member of the VEGFxxxb family to be identified and was discovered in human renal cortex [62]. This isoform is expressed early in human kidney embryonic development and is expressed endogenously in differentiated, but not dedifferentiated human podocytes [64]. Schumacher et al. demonstrated that in normal renal development, the capillary loop stage showed the highest VEGF165 expression that diminishes afterward with a switch in favor of the VEGF165b isoform, which showed its highest expression in adult control glomeruli [65]. Several reports have shown that the inclusion of exon $8 \mathrm{a}$ results in the conventional proangiogenic and propermeability of VEGFxxx family but replacement of $8 \mathrm{a}$ with exon $8 \mathrm{~b}$ in the VEGFxxxb family produces peptides that are antiangiogenic, inhibits permeability chronically, and reduces rather than promotes tumor growth [66-68]. In renal-cell [62], prostate [69], colon carcinoma [70], and malignant melanoma [71], VEGF165b has been reported to be downregulated. A dysregulation of VEGFxxxb expression was also observed in the glomeruli of humans with DenysDrash syndrome [65] and in human preeclamptic placentas [72].

In addition to the renal lesion reported in preeclampsia, altered levels of VEGF-A protein and/or mRNA were detected in various human and rodent experimental renal disease models associated with proteinuria. Diabetic nephropathy is characterized by the development of proteinuria followed by decreased glomerular filtration in association with glomerulosclerosis. Researchers have observed that urinary VEGF $[73,74]$, renal and/or glomerular VEGF levels and VEGF receptors are consistently increased in diverse experimental models of diabetes, especially early in the course of the disorder $[75,76]$. In vitro, exposure of cultured podocytes to high glucose concentrations also upregulates VEGF expression [77]. Blocking the increased VEGF-A with
anti-pan-VEGF antibodies improves the diabetes-related early renal dysfunction $[73,78]$. Consequently, the inhibition of upregulated VEGF and its receptors resulted in beneficial effects on diabetes-induced functional and structural alterations.

Several types of glomerulonephritis, in which experimental models or human kidney biopsies were analyzed, showed contradictory results. Glomerulonephritis (GN) are inflammatory glomerular diseases associated to proteinuria, frequently leading to chronic disease progression and renal replacement therapy. In some experimental models of human minimal change nephropathy (MCN), VEGF and its receptors were, as in diabetic nephropathy, upregulated and correlated with the severity of proteinuria [79]. In patients with MCN and nephrotic syndrome, the urinary VEGF levels are increased and positively correlated with the degree of proteinuria [80]. In situ hybridization studies revealed that VEGF mRNA expression was upregulated in podocytes with MCN [81]. Patients with congenital nephrotic syndrome of the Finnish type showed a slightly increased VEGF expression in glomeruli [82]. Recently, Hohenstein et al. have found increased glomerular VEGF expression in biopsies from patients with endocapillary nephritis, membranoproliferative glomerulonephritis (MPGN), and crescentic nephritis [83]. Biopsies from patients with membranous glomerulonephritis (MGN) presented markedly increased VEGF protein in podocytes [83]. Depending on the degree of the injury and inflammation in the glomeruli with glomerulosclerosis, VEGFR-1 expression was increased on various cell types in focal areas, and VEGFR-2 expression was more prominent in podocytes of biopsies with GN [83]. In contrast, other studies found either a reduction or lack of changes in the VEGF-VEGFR system in experimental or clinical studies of MGN [81, 84], MPGN [85, 86], crescentic glomerulonephritis [87], or focal segmental glomerulosclerosis (FSGS) [76]. Müller-Deile et al. reported a clinical case with metastatic cancers, where after total tumour nephrectomy, the patient developed FSGS and podocyturia in association with tyrosine kinase inhibitor treatments [88]. The downregulation of VEGF expression was also reported in adriamycin nephropathy rats [89] and puromycin aminonucleoside rats [90] with the progressive aggravation of foot processes morphology and development of proteinuria. These results suggest that VEGF might exert multiple effects on the glomerular pathophysiologic processes and that the expression balance of VEGF is essential for preserving the normal glomerular filtration function.

To demonstrate that VEGF-A signaling is a key for proper maintenance of the glomerular filtration barrier, several animal studies have been performed. Eremina et al. generated mice with gain or loss of function of VEGF specifically in the podocyte [91], avoiding the embryolethal effects observed in VEGF-A knockout [92] and in haploinsufficient mice [93]. Mice with podocyte-specific deletion of both VEGF alleles died at birth or within 18 hours of birth with kidney failure and grossly abnormal glomeruli. The glomeruli of these mice fail to form functional filtration barriers due to major defects in endothelial cell migration, survival, and differentiation. Mice with podocyte-specific 
heterozygosity for VEGF developed proteinuria, endotheliosis, and bloodless glomeruli, characteristic findings in patients with preeclampsia, which progressed to nephrotic syndrome and followed by kidney failure [91]. Kitamoto and colleagues administered VEGF-blocking antibodies to murine pups and observed that the development of the kidney was impaired in terms of glomerulogenesis and nephrogenesis [94]. Conversely, the overexpression of VEGF$A$ in podocytes also resulted in a dramatic phenotype due to a collapsing glomerulopathy and end-stage renal failure [91]. All these discoveries emphasize the role of VEGF-A in renal glomeruli and demonstrate that a tight regulation of VEGFA signaling is required for development and maintenance of the glomerular filtration barrier.

Different groups have examined the expression of slit diaphragm proteins in acquired glomerular diseases where the VEGF levels are altered. In both experimental and human diabetes, one of the several diseases involving a podocyte injury, various groups have found nephrin in the urine, downregulation of nephrin expression in the slit diaphragm and decreased nephrin mRNA levels that inversely correlated with the degree of proteinuria [95-98]. Moreover, no changes in the expression of CD2AP and podocin in podocytes from diabetic patients were observed [97]. However, reduction of the elevated concentrations of glycated albumin in diabetes animals showed significant reduction of proteinuria and restoration of distorted glomerular nephrin and VEGF expression [99]. Chun-Liang et al. demonstrated that activation of Notch-1 signaling in high glucose (HG)treated human podocytes and in the glomeruli of diabetic rats produces increased VEGF expression, nephrin downregulation, and increased apoptosis, while the inhibition of Notch-1 signaling significantly abrogated VEGF activation, nephrin repression, and ameliorated proteinuria in HGstressed cells and in diabetic kidney [100]. Garovic et al. reported that proteinuria in patients with preeclampsia is associated with downregulation of podocyte foot process proteins, as nephrin and synaptopodin [101]. Koop et al. and Fukuda et al. reported reduced nephrin expression at protein and/or mRNA level in patients and animals with MCN, and a significant decrease in the expression of podocin, podocalyxin and CD2AP was also seen in MCN [102-104]. Nevertheless, the genetic analyses of patients with MCN revealed heterozygous amino acid changes in nephrin and podocin, but no amino acid substitutions were detected in Neph1 and CD2AP genes $[105,106]$. On the other hand, several reports have investigated the expression of nephrin in others glomerulosclerosis diseases, which were not always consistent. Furness et al. [107], Huh et al. [108], Srivastava et al. [109], Wang et al. [110], and Doublier et al. [111] reported a downregulation in nephrin expression in patients with focal segmental glomerular sclerosis, MPGN and membranous nephropathy ( $\mathrm{MN})$, whereas, as reported by Patrakka et al. [112], Guan et al. [113], and Hingorani et al. [114], no significant changes were seen in expression of nephrin at all. Hara et al. showed that blockage of VEGF activity in rats with progressive GN resulted in massive urinary protein excretion and downregulated expression of nephrin [115]. No extensive changes in the expression of others slit diaphragm proteins were observed in glomerulosclerosis [116, 117]. These discrepant results in some nephrotic syndromes may be attributed to differences in the degree of glomerulosclerosis and the methods used for detecting protein expression. Clearly, these studies demonstrate that disruption in VEGF levels are not only associated with increased proteinuria but also with readjustment of nephrin-dependent slit diaphragm structure. VEGF-nephrin signaling in podocytes may act through one or more survival signaling pathways, but these pathways are not full known.

\section{Paracrine or Autocrine Podocytic VEGF-VEGFR System?}

During fetal development, the podocytes are one of the cell types producing the largest amounts of VEGF-A. When podocytes are fully differentiated, they continue to express VEGF-A, even though absolute levels of expression decrease [118]. The adjacent and nearby associated glomerular endothelial cells express VEGFR-1 and VEGFR-2 tyrosine kinase receptors $[42,91]$. As a consequence, some authors have speculated that in normal conditions, a paracrine regulatory mechanism where the podocyte-secreted VEGF can bind to VEGF receptors on mesangium or glomerular endothelial cells. It is now broadly accepted that VEGF must move in the opposite direction of the glomerular filtrate in order to bind to its receptors. This notion is supported by the following studies. Quaggin group postulated that since podocytes produce the constitutive highest levels of VEGF, there is a concentration gradient favoring diffusion of VEGF from the podocyte to the glomerular endothelial cells in addition to the finding that the diffusion rate across the glomerular basement membrane is the predominant transport mechanism for solutes with the molecular radii under $30 \AA$ while that of VEGF is about $26 \AA[45,119]$.

Sison et al. [120] have found that induced wholebody postnatal deletion of VEGFR-2 caused marked damage in glomerular microvascular beds with loss of viable endothelial cells, whereas the same authors have observed nonthrombotic microangiopathy effect in the glomeruli of mice with whole body postnatal deletion of VEGFR-1 [120]. Eremina et al. showed that genetic inhibition of VEGF in mature podocytes also resulted in glomerular endothelial cell damage and thrombotic microangiopathy [45]. In addition, Sison et al. observed that the deletion of VEGFR-2 specifically from podocytes never developed proteinuria or glomerular injury, and the glomerular filtration barrier was intact [120]. These observations support the idea that VEGF signaling through VEGFR-2, not through VEGFR-1, is essential for maintaining the glomerular filtration barrier via a paracrine mechanism.

Nephrin cytoplasmic domain has a series of conserved tyrosine-based motifs [18], which are phosphorylated by Fyn kinase and are able to bind and phosphorylate the Nck adaptor [19] and the cytoplasmic kinase phosphatidylinositol3-OH kinase (PI3K) proteins via their SH2 domains [20]. Phosphorylation of Nck contributes to the reorganization of the actin cytoskeleton in podocytes [21], and phosphorylated PI3K stimulates the serine-threonine kinase AKT 
signaling, leading the phosphorylation of molecules involve in the prevention of apoptosis and safeguard viability in podocytes [20]. Interestingly, Foster et al. postulated that VEGF treatment caused reduction of apoptosis through nephrin phosphorylation together with a decrease in AKTsignaling [121]. Sugimoto et al. observed that a single intravenous infusion of anti-VEGF antibodies into normal healthy mice produced excessive albumin excretion in the urine, massive glomerular, endothelial cell damage, and significantly reduction of nephrin expression [49]. Also, Foster et al. provided the first evidence that in podocytes, VEGF is associated with the cell membrane in normal human glomeruli [121]. This phenomenon could be explained either by an accumulation of VEGF protein before secretion or by the sequestration of VEGF, via receptor binding, onto the podocyte cell surface [122]. To answer these issues, the same group observed that the addition of type III receptor tyrosine-kinase inhibitor abolished the reducing cell death induced by VEGF, suggesting that a tyrosine kinasemediated receptor for VEGF-A exists on podocytes [122]. These results suggest that podocytes may have the potential to bind the VEGF that they secrete. This hypothesis is also supported by other independent groups. They observed that, using transmission electron microscopy, VEGFR-2 is expressed in mice podocytes in vivo $[123,124]$. Guan et al. observed that VEGFR-2 mRNA levels are increased in mouse-differentiated cultured podocytes [125], and recombinant VEGF 165 induced VEGFR-2 mRNA and protein levels and reduced apoptosis in these differentiated podocytes. In contrast, VEGFR-2 was not detected in conditionally immortalized human podocytes [122]. These discrepancies may be species related. Nonetheless, Müller-Deile et al. identified that VEGFR-2 is the main receptor responsible for the PI3K/AKT regulation and podocyte survival in response to autocrine levels of VEGF-A and VEGF-C in human podocytes in vitro [126]. VEGF-A signaling also showed the ability to regulate slit diaphragm proteins by inducing podocin upregulation and increasing its interaction with CD2AP in vitro [125]. We have recently demonstrated that transgenic mice overexpressing $\mathrm{VEGF}_{164}$ in podocyte showed proteinuria, glomerular basement membrane thickening, loss of slit diaphragms, podocyte effacement, induced VEGFR-2 phosphorylation, and downregulation of nephrin expression [123]. Moreover, we provided the first evidence that nephrin, a podocyte-specific protein, and VEGFR-2 are associated in vivo, suggesting the expression of VEGFR2 in podocytes [123]. Podocyte-derived VEGF has a welldocumented paracrine function on endothelial cells as well as an autocrine function on podocytes themselves. However, several groups disagree with the idea of podocyte-VEGFR2 mediating the autocrine function of VEGF. Nevertheless, all these data indicate not only that podocytes possess a functional autocrine VEGF-A signaling through VEGFR-2 but also that VEGF-A signaling regulates the expression of slit diaphragm proteins. Additionally, our studies do not exclude the possible effects of podocyte $\mathrm{VEGF}_{164}$ overexpression through VEGFR-1, which is able to regulate in a negative fashion the activity of VEGF and thereby preventing VEGF binding to VEGFR-2.
Although the podocyte has been described to be the site of VEGF production within the glomerulus, the role and/or interaction of podocyte-derived VEGF-A in glomerular health and disease still remain debatable. In view of the fact that VEGF receptors (VEGFR-1 and VEGFR-2) are expressed at the highest levels on glomerular endothelial cells, investigations have primarily focused on the paracrine functions for podocyte-derived VEGF-A. At present, several studies demonstrated that VEGFR-2 is expressed at low levels by podocytes and highlight the existence and importance of autocrine podocytic VEGF-VEGFR system in normal and injured podocytes. Disruption or an imbalance of podocytederived VEGF-A produces changes in slit diaphragm proteins, and, as consequence, it leads to podocyte effacement and proteinuria. Loss or failure of podocytes function contributes to the development of glomerulosclerosis, which is the final stage of various renal diseases.

The biology of the VEGF-VEGFR system is indeed complex. Any manipulation of this system has to be cautious in order not to disrupt its fragile balance. To find a therapeutic strategy is going to be challenging, especially considering the vast diversity of VEGF isoforms and because specific compounds interfering with the VEGF-VEGFR system are limited. Although data is currently unavailable in humans, several strategies to either inhibit or enhance the VEGF axis have shown promising results in animal models of renal disease.

\section{Acknowledgment}

The author would like to thank Dr. Delma Veron for critical review of this manuscript.

\section{References}

[1] B. Haraldsson, J. Nystrom, and W. M. Deen, "Properties of the glomerular barrier and mechanisms of proteinuria," Physiological Reviews, vol. 88, no. 2, pp. 451-487, 2008.

[2] M. Jeansson and B. Haraldsson, "Morphological and functional evidence for an important role of the endothelial cell glycocalyx in the glomerular barrier," American Journal of Physiology, vol. 290, no. 1, pp. F111-F116, 2006.

[3] D. R. Abrahamson, "Structure and development of the glomerular capillary wall and basement membrane," American Journal of Physiology, vol. 253, no. 2, pp. F783-F794, 1987.

[4] T. Pihlajaniemi, "Molecular properties of the glomerular basement membrane," Contributions to Nephrology, vol. 117, pp. 46-79, 1996.

[5] R. Timpl and J. C. Brown, "Supramolecular assembly of basement membranes," Bioessays, vol. 18, no. 2, pp. 123-132, 1996.

[6] J. Reiser, W. Kriz, M. Kretzler, and P. Mundel, "The glomerular slit diaphragm is a modified adherens junction," Journal of the American Society of Nephrology, vol. 11, no. 1, pp. 1-8, 2000.

[7] D. Kerjaschki, D. J. Sharkey, and M. G. Farquhar, "Identification and characterization of podocalyxin, the major sialoprotein of the renal glomerular epithelial cell," Journal of Cell Biology, vol. 98, no. 4, pp. 1591-1596, 1984. 
[8] J. A. Kreidberg, M. J. Donovan, S. L. Goldstein et al., " $\alpha 3 \beta 1$ integrin has a crucial role in kidney and lung organogenesis," Development, vol. 122, no. 11, pp. 3537-3547, 1996.

[9] C. J. Raats, J. van den Born, M. A. Bakker et al., "Expression of agrin, dystroglycan, and utrophin in normal renal tissue and in experimental glomerulopathies," American Journal of Pathology, vol. 156, no. 5, pp. 1749-1765, 2000.

[10] D. Drenckhahn and R. F. Franke, "Ultrastructural organization of contractile and cytoskeletal proteins in glomerular podocytes of chicken, rat, and man," Laboratory Investigation, vol. 59, no. 5, pp. 673-682, 1988.

[11] Y. S. Kanwar, Z. Z. Liu, N. Kashihara, and E. I. Wallner, "Current status of the structural and functional basis of glomerular filtration and proteinuria," Seminars in Nephrology, vol. 11, no. 4, pp. 390-413, 1991.

[12] M. Kestilä, U. Lenkkeri, M. Männikkö et al., "Positionally cloned gene for a novel glomerular protein-nephrin-is mutated in congenital nephrotic syndrome," Molecular Cell, vol. 1, no. 4, pp. 575-582, 1998.

[13] V. Ruotsalainen, P. Ljungberg, J. Wartiovaara et al., "Nephrin is specifically located at the slit diaphragm of glomerular podocytes," Proceedings of the National Academy of Sciences of the United States of America, vol. 6, no. 14, pp. 7962-7967, 1999.

[14] K. Tryggvason, "Unraveling the mechanisms of glomerular ultrafiltration: nephrin, a key component of the slit diaphragm," Journal of the American Society of Nephrology, vol. 10, no. 11, pp. 2440-2445, 1999.

[15] K. Yan, J. Khoshnoodi, V. Ruotsalainen, and K. Tryggvason, "N-linked glycosylation is critical for the plasma membrane localization of nephrin," Journal of the American Society of Nephrology, vol. 13, no. 5, pp. 1385-1389, 2002.

[16] P. Gerke, T. B. Huber, L. Sellin, T. Benzing, and G. Walz, "Homodimerization and heterodimerization of the glomerular podocyte proteins nephrin and NEPH1," Journal of the American Society of Nephrology, vol. 14, no. 4, pp. 918-926, 2003.

[17] J. Wartiovaara, L. G. Ofverstedt, J. Khoshnoodi et al., "Nephrin strands contribute to a porous slit diaphragm scaffold as revealed by electron tomography," Journal of Clinical Investigation, vol. 114, no. 10, pp. 1475-1483, 2004.

[18] H. Putaala, K. Sainio, H. Sariola, and K. Tryggvason, "Primary structure of mouse and rat nephrin cDNA and structure and expression of the mouse gene," Journal of the American Society of Nephrology, vol. 11, no. 6, pp. 991-1001, 2000.

[19] R. Verma, B. Wharram, I. Kovari et al., "Fyn binds to and phosphorylates the kidney slit diaphragm component nephrin," Journal of Biological Chemistry, vol. 278, no. 23, pp. 20716-20723, 2003.

[20] T. B. Huber, B. Hartleben, J. Kim et al., "Nephrin and CD2AP associate with phosphoinositide 3-OH kinase and stimulate AKT-dependent signaling," Molecular and Cellular Biology, vol. 23, no. 14, pp. 4917-4928, 2003.

[21] N. Jones, I. M. Blasutig, V. Eremina et al., "Nck adaptor proteins link nephrin to the actin cytoskeleton of kidney podocytes," Nature, vol. 6, no. 7085, pp. 818-823, 2006.

[22] H. Putaala, R. Soininen, P. Kilpeläinen, J. Wartiovaara, and K. Tryggvason, "The murine nephrin gene is specifically expressed in kidney, brain and pancreas: inactivation of the gene leads to massive proteinuria and neonatal death," Human Molecular Genetics, vol. 10, no. 1, pp. 1-8, 2001.

[23] H. Holthöfer, H. Ahola, M. L. Solin et al., "Nephrin localizes at the podocyte filtration slit area and is characteristically spliced in the human kidney," American Journal of Pathology, vol. 155, no. 5, pp. 1681-1687, 1999.

[24] J. Patrakka, M. Kestilä, J. Wartiovaara et al., "Congenital nephrotic syndrome (NPHS1): features resulting from different mutations in finnish patients," Kidney International, vol. 58, no. 3, pp. 972-980, 2000.

[25] C. Holmberg, M. Antikainen, K. Rönnholm, M. AlaHouhala, and H. Jalanko, "Management of congenital nephrotic syndrome of the finnish type," Pediatric Nephrology, vol. 9, no. 1, pp. 87-93, 1995.

[26] T. Inoue, E. Yaoita, H. Kurihara et al., "FAT is a component of glomerular slit diaphragms," Kidney International, vol. 59, no. 3, pp. 1001-1012, 2001.

[27] E. Schnabel, M. Anderson, and M. G. Farquhar, "The tight junction protein $\mathrm{ZO}-1$ is concentrated along slit diaphragms of the glomerular epithelium," Journal of Cell Biology, vol. 111, no. 3, pp. 255-263, 1990.

[28] N. Y. Shi, J. Li, R. Cotran, P. Mundel, J. H. Miner, and A. S. Shaw, "CD2AP localizes to the slit diaphragm and binds to nephrin via a novel C-terminal domain," American Journal of Pathology, vol. 159, no. 6, pp. 2303-2308, 2001.

[29] N. Y. Shih, J. Li, V. Karpitskii et al., "Congenital nephrotic syndrome in mice lacking CD2-associated protein," Science, vol. 286, no. 5438, pp. 312-315, 1999.

[30] K. Schwarz, M. Simons, J. Reiser et al., "Podocin, a raftassociated component of the glomerular slit diaphragm, interacts with CD2AP and nephrin," Journal of Clinical Investigation, vol. 108, no. 11, pp. 1621-1629, 2001.

[31] T. B. Huber, M. Köttgen, B. Schilling, G. Walz, and T. Benzing, "Interaction with Podocin facilitates nephrin signaling," Journal of Biological Chemistry, vol. 276, no. 45, pp. 4154341546, 2001.

[32] S. Lehtonen, F. Zhao, and E. Lehtonen, "CD2-associated protein directly interacts with the actin cytoskeleton," American Journal of Physiology, vol. 283, no. 4, pp. F734-F743, 2002.

[33] L. Sellin, T. B. Huber, P. Gerke, I. Quack, H. Pavenstädt, and G. Walz, "NEPH1 defines a novel family of podocin interacting proteins," FASEB Journal, vol. 17, no. 1, pp. 115117, 2003.

[34] T. B. Huber, M. Schmidts, P. Gerke et al., "The carboxyl terminus of neph family members binds to the PDZ domain protein zonula occludens-1," Journal of Biological Chemistry, vol. 278, no. 15, pp. 13417-13421, 2003.

[35] J. Reiser, K. R. Polu, C. C. Möller et al., "TRPC6 is a glomerular slit diaphragm-associated channel required for normal renal function," Nature Genetics, vol. 37, no. 7, pp. 739-744, 2005.

[36] D. B. Donoviel, D. D. Freed, H. Vogel et al., "Proteinuria and perinatal lethality in mice lacking NEPH1, a novel protein with homology to NEPHRIN," Molecular and Cellular Biology, vol. 21, no. 14, pp. 4829-4836, 2001.

[37] N. Boute, O. Gribouval, S. Roselli et al., "NPHS2, encoding the glomerular protein podocin, is mutated in autosomal recessive steroid-resistant nephrotic syndrome," Nature Genetics, vol. 24, no. 4, pp. 349-354, 2000.

[38] L. Ciani, A. Patel, N. D. Allen, and C. Ffrench-Constant, "Mice lacking the giant protocadherin mFAT1 exhibit renal slit junction abnormalities and a partially penetrant cyclopia and anophthalmia phenotype," Molecular and Cellular Biology, vol. 23, no. 10, pp. 3575-3582, 2003.

[39] S. J. Shankland, “The podocyte's response to injury: role in proteinuria and glomerulosclerosis," Kidney International, vol. 69, no. 12, pp. 2131-2147, 2006. 
[40] J. M. Kaplan, S. H. Kim, K. N. North et al., "Mutations in ACTN4, encoding $\alpha$-actinin- 4 , cause familial focal segmental glomerulosclerosis," Nature Genetics, vol. 24, no. 3, pp. 251256, 2000.

[41] R. Doyonnas, D. B. Kershaw, C. Duhme et al., "Anuria, omphalocele, and perinatal lethality in mice lacking the CD34-related protein podocalyxin," Journal of Experimental Medicine, vol. 194, no. 1, pp. 13-27, 2001.

[42] V. Eremina, H. J. Baelde, and S. E. Quaggin, "Role of the VEGF - a signaling pathway in the glomerulus: evidence for crosstalk between components of the glomerular filtration barrier," Nephron Physiology, vol. 106, no. 2, pp. p32-p37, 2007.

[43] J. C. Yang, L. Haworth, R. M. Sherry et al., "A randomized trial of bevacizumab, an anti-vascular endothelial growth factor antibody, for metastatic renal cancer," New England Journal of Medicine, vol. 349, no. 5, pp. 427-434, 2003.

[44] F. Kabbinavar, H. I. Hurwitz, L. Fehrenbacher et al., "Phase II, randomized trial comparing bevacizumab plus fluorouracil (FU)/leucovorin (LV) with FU/LV alone in patients with metastatic colorectal cancer," Journal of Clinical Oncology, vol. 21, no. 1, pp. 60-65, 2003.

[45] V. Eremina, J. A. Jefferson, J. Kowalewska et al., "VEGF inhibition and renal thrombotic microangiopathy," New England Journal of Medicine, vol. 358, no. 11, pp. 1129-1136, 2008.

[46] T. Patel, J. Morgan, G. Demetri et al., "A preeclampsialike syndrome characterized by reversible hypertension and proteinuria induced by the multitargeted kinase inhibitors sunitinib and sorafenib," Journal of the National Cancer Institute, vol. 100, no. 4, pp. 282-284, 2008.

[47] S. E. Maynard, J. Y. Min, J. Merchan et al., "Excess placental soluble fms-like tyrosine kinase 1 (sFlt1) may contribute to endothelial dysfunction hypertension, and proteinuria in preeclampsia," Journal of Clinical Investigation, vol. 111, no. 5, pp. 649-658, 2003.

[48] J. Müller-Deile and M. Schiffer, "Renal involvement in preeclampsia: similarities to VEGF ablation therapy," Journal of Pregnancy, vol. 2011, Article ID 176973, 6 pages, 2011.

[49] H. Sugimoto, Y. Hamanog, D. Charytan et al., "Neutralization of circulating vascular endothelial growth factor (VEGF) by anti-VEGF antibodies and soluble VEGF receptor 1 (sFlt1) induces proteinuria," Journal of Biological Chemistry, vol. 278, no. 15, pp. 12605-12608, 2003.

[50] G. Neufeld, T. Cohen, S. Gengrinovitch, and Z. Poltorak, "Vascular endothelial growth factor (VEGF) and its receptors," FASEB Journal, vol. 13, no. 1, pp. 9-22, 1999.

[51] M. Simon, H. J. Gröne, O. Jöhren et al., "Expression of vascular endothelial growth factor and its receptors in human renal ontogenesis and in adult kidney," American Journal of Physiology, vol. 268, no. 2, pp. F240-F250, 1995.

[52] M. Simon, W. Rockl, C. Hornig et al., "Receptors of vascular endothelial growth factor/vascular permeability factor (VEGF/VPF) in fetal and adult human kidney: localization and [125I]VEGF binding sites," Journal of the American Society of Nephrology, vol. 9, no. 6, pp. 1032-1044, 1998.

[53] K. Kondo, S. Hiratsuka, E. Subbalakshmi, H. Matsushime, and M. Shibuya, "Genomic organization of the flt-1 gene encoding for vascular endothelial growth factor (VEGF) Receptor-1 suggests an intimate evolutionary relationship between the 7-Ig and the 5-Ig tyrosine kinase receptors," Gene, vol. 208, no. 2, pp. 297-305, 1998.

[54] M. Shibuya, S. Yamaguchi, A. Yamane et al., "Nucleotide sequence and expression of a novel human receptor-type tyrosine kinase gene (flt) closely relatd to the fms family," Oncogene, vol. 8, no. 4, pp. 519-524, 1990.

[55] B. I. Terman, M. E. Carrion, E. Kovacs, B. A. Rasmussen, R. L. Eddy, and T. B. Shows, "Identification of a new endothelial cell growth factor receptor tyrosine kinase," Oncogene, vol. 6, no. 9, pp. 1677-1683, 1991.

[56] J. Schlessinger, "Cell signaling by receptor tyrosine kinases," Cell, vol. 103, no. 2, pp. 211-225, 2000.

[57] E. Sulpice, J. Plouët, M. Bergé, D. Allanic, G. Tobelem, and T. Merkulova-Rainon, "Neuropilin-1 and neuropilin-2 act as coreceptors, potentiating proangiogenic activity," Blood, vol. 111, no. 4, pp. 2036-2045, 2008.

[58] Y. Shaked, G. Bocci, R. Munoz et al., "Cellular and molecular surrogate markers to monitor targeted and non-targeted antiangiogenic drug activity and determine optimal biologic dose," Current Cancer Drug Targets, vol. 5, no. 7, pp. 551-559, 2005.

[59] R. L. Kendall and K. A. Thomas, "Inhibition of vascular endothelial cell growth factor activity by an endogenously encoded soluble receptor," Proceedings of the National Academy of Sciences of the United States of America, vol. 90, no. 22, pp. 10705-10709, 1993.

[60] J. E. Park, H. H. Chen, J. Winer, K. A. Houck, and N. Ferrara, "Placenta growth factor. potentiation of vascular endothelial growth factor bioactivity, in vitro and in vivo, and high affinity binding to Flt-1 but not to Flk-1/KDR," Journal of Biological Chemistry, vol. 269, no. 41, pp. 25646-25654, 1994.

[61] K. A. Houck, N. Ferrara, J. Winer, G. Cachianes, B. Li, and D. W. Leung, "The vascular endothelial growth factor family: identification of a fourth molecular species and characterization of alternative splicing of RNA," Molecular Endocrinology, vol. 5, no. 12, pp. 1806-1814, 1991.

[62] D. Bates, T. Cui, J. Doughty et al., "VEGF165b, an inhibitory splice variant of vascular endothelial growth factor, is downregulated in renal cell carcinoma," Cancer Research, vol. 62, no. 14, pp. 4123-4131, 2002.

[63] H. Kawamura, X. Li, K. Goishi et al., "Neuropilin-1 in regulation of VEGF-induced activation of p38MAPK and endothelial cell organization," Blood, vol. 112, no. 9, pp. 3638-3649, 2008.

[64] T. Cui, R. Foster, M. Saleem et al., "Differentiated human podocytes endogenously express an inhibitory isoform of vascular endothelial growth factor (VEGF165b) mRNA and protein," American Journal of Physiology, vol. 286, no. 4, pp. F767-F773, 2004.

[65] V. A. Schumacher, S. Jeruschke, F. Eitner et al., "Impaired glomerular maturation and lack of VEGF165b in DenysDrash syndrome," Journal of the American Society of Nephrology, vol. 18, no. 3, pp. 719-729, 2007.

[66] H. Bevan, N. van den Akker, Y. Qiu et al., "The alternatively spliced anti-angiogenic family of VEGF isoforms VEGF xxxb in human kidney development," Nephron Physiology, vol. 110, no. 4, pp. p57-p67, 2008.

[67] S. Cébe Suarez, M. Pieren, L. Cariolato et al., "A VEGF-A splice variant defective for heparan sulfate and neuropilin1 binding shows attenuated signaling through VEGFR-2," Cellular and Molecular Life Sciences, vol. 63, no. 17, pp. 20672077, 2006.

[68] S. J. Harper and D. Bates, "VEGF-A splicing: the key to antiangiogenic therapeutics?" Nature Reviews Cancer, vol. 8, no. 11 , pp. 880-887, 2008.

[69] J. Woolard, W. Wang, H. Bevan et al., "VEGF165b, an inhibitory vascular endothelial growth factor splice variant: mechanism of action, in vivo effect on angiogenesis and 
endogenous protein expression," Cancer Research, vol. 64, no. 21, pp. 7822-7835, 2004.

[70] A. Varey, E. Rennel, Y. Qiu et al., "VEGF165b, an antiangiogenic VEGF-A isoform, binds and inhibits bevacizumab treatment in experimental colorectal carcinoma: balance of pro- and antiangiogenic VEGF-A isoforms has implications for therapy," British Journal of Cancer, vol. 98, no. 8, pp. 1366-1379, 2008.

[71] R. Pritchard-Jones, D. Dunn, Y. Qiu et al., "Expression of VEGFxxxb, the inhibitory isoforms of VEGF, in malignant melanoma," British Journal of Cancer, vol. 97, no. 2, pp. 223 230, 2007.

[72] D. Bates, P. MacMillan, J. Manjaly et al., "The endogenous anti-angiogenic family of splice variants of VEGF, VEGF $\mathrm{xxxb}$, are down-regulated in pre-eclamptic placentae at term," Clinical Science, vol. 110, no. 5, pp. 575-585, 2006.

[73] N. H. Kim, J. H. Oh, J. A. Seo et al., "Vascular endothelial growth factor (VEGF) and soluble VEGF receptor FLT-1 in diabetic nephropathy," Kidney International, vol. 67, no. 1, pp. 167-177, 2005.

[74] D. R. Cha, Y. S. Kang, S. Y. Han et al., "Vascular endothelial growth factor is increased during early stage of diabetic nephropathy in type II diabetic rats," Journal of Endocrinology, vol. 183, no. 1, pp. 183-194, 2004.

[75] M. E. Cooper, D. Vranes, S. Youssef et al., "Increased renal expression of vascular endothelial growth factor (VEGF) and its receptor VEGFR-2 in experimental diabetes," Diabetes, vol. 48, no. 11, pp. 2229-2239, 1999.

[76] B. F. Schrijvers, A. Flyvbjerg, and A. S. De Vriese, "The role of vascular endothelial growth factor (VEGF) in renal pathophysiology," Kidney International, vol. 65, no. 6, pp. 2003-2017, 2004.

[77] S. Chen, J. S. Lee, M. C. Iglesias-de la Cruz et al., "Angiotensin II stimulates $\alpha 3$ (IV) collagen production in mouse podocytes via TGF- $\beta$ and VEGF signalling: implications for diabetic glomerulopathy," Nephrology Dialysis Transplantation, vol. 20, no. 7, pp. 1320-1328, 2005.

[78] B. F. Schrijvers, A. Flyvbjerg, R. G. Tilton, N. H. Lameire, and A. S. De Vriese, "A neutralizing VEGF antibody prevents glomerular hypertrophy in a model of obese type 2 diabetes, the Zucker diabetic fatty rat," Nephrology Dialysis Transplantation, vol. 21, no. 2, pp. 324-329, 2006.

[79] Y. Horita, M. Miyazaki, T. Koji et al., "Expression of vascular endothelial growth factor and its receptors in rats with protein-overload nephrosis," Nephrology Dialysis Transplantation, vol. 13, no. 10, pp. 2519-2528, 1998.

[80] K. Matsumoto and K. Kanmatsuse, "Elevated vascular endothelial growth factor levels in the urine of patients with minimal-change nephrotic syndrome," Clinical Nephrology, vol. 55, no. 4, pp. 269-274, 2001.

[81] E. Bailey, M. J. Bottomley, S. Westwell et al., "Vascular endothelial growth factor mRNA expression in minimal change, membranous, and diabetic nephropathy demonstrated by non-isotopic in situ hybridisation," Journal of Clinical Pathology, vol. 52, pp. 735-738, 1999.

[82] A. Kaukinen, A. Kuusniemi, I. Lautenschlager, and H. Jalanko, "Glomerular endothelium in kidneys with congenital nephrotic syndrome of the finnish type (NPHS1)," Nephrology Dialysis Transplantation, vol. 23, no. 4, pp. 1224 1232, 2008.

[83] B. Hohenstein, M. Colin, C. Foellmer et al., "Autocrine VEGF-VEGF-R loop on podocytes during glomerulonephritis in humans," Nephrology Dialysis Transplantation, vol. 25, no. 10 , pp. 3170-3180, 2010.
[84] E. Honkanen, A. Teppo, and C. Gronhagen-Riska, "Decreased urinary excretion of vascular endothelial growth factor in idiopathic membranous glomerulonephritis," Kidney International, vol. 57, no. 6, pp. 2343-2349, 2000.

[85] A. Kubo, Y. Nishitani, N. Minamino et al., "Adrenomedullin gene transcription is decreased in peripheral blood mononuclear cells of patients with IgA nephropathy," Nephron, vol. 85, no. 3, pp. 201-206, 2000.

[86] A. Amore, G. Conti, P. Cirina et al., "Aberrantly glycosylated IgA molecules downregulate the synthesis and secretion of vascular endothelial growth factor in human mesangial cells," American Journal of Kidney Diseases, vol. 36, no. 6, pp. 12421252, 2000.

[87] H. T. Yuan, P. G. Tipping, X. Z. Li, D. A. Long, and A. S. Woolf, "Angiopoietin correlates with glomerular capillary loss in anti-glomerular basement membrane glomerulonephritis," Kidney International, vol. 61, no. 6, pp. 20782089, 2002.

[88] J. Müller-Deile, V. Bröcker, V. Grünwald et al., "Renal side effects of VEGF-blocking therapy,” NDT Plus, vol. 3, no. 2, pp. 172-175, 2010.

[89] Q. Fan, Y. Xing, J. Ding, and N. Guan, "Reduction in VEGF protein and phosphorylated nephrin associated with proteinuria in adriamycin nephropathy rats," Nephron, vol. 111, no. 4, pp. e92-e102, 2009.

[90] L. Fan, T. Wakayama, S. Yokoyama, O. Amano, and S. Iseki, "Downregulation of vascular endothelial growth factor and its receptors in the kidney in rats with puromycin aminonucleoside nephrosis," Nephron, vol. 90, no. 1, pp. 95$102,2002$.

[91] V. Eremina, M. Sood, J. Haigh et al., "Glomerular-specific alterations of VEGF-A expression lead to distinct congenital and acquired renal diseases," Journal of Clinical Investigation, vol. 111, no. 5, pp. 707-716, 2003.

[92] N. Ferrara, K. Carver-Moore, H. Chen et al., "Heterozygous embryonic lethality induced by targeted inactivation of the VEGF gene," Nature, vol. 380, no. 6573, pp. 439-442, 1996.

[93] P. Carmeliet, V. Ferreira, G. Breier et al., "Abnormal blood vessel development and lethality in embryos lacking a single VEGF allele," Nature, vol. 380, no. 6573, pp. 435-439, 1996.

[94] Y. Kitamoto, H. Tokunaga, and K. Tomita, "Vascular endothelial growth factor is an essential molecule for mouse kidney development: glomerulogenesis and nephrogenesis," Journal of Clinical Investigation, vol. 99, no. 10, pp. 23512357, 1997.

[95] P. Aaltonen, P. Luimula, E. Åström et al., "Changes in the expression of nephrin gene and protein in experimental diabetic nephropathy," Laboratory Investigation, vol. 81, no. 9, pp. 1185-1190, 2001.

[96] S. Doublier, G. Salvidio, E. Lupia et al., "Nephrin expression is reduced in human diabetic nephropathy," Diabetes, vol. 51, no. 4, pp. 1023-1030, 2003.

[97] A. Benigni, E. Gagliardini, S. Tomasoni et al., "Selective impairment of gene expression and assembly of nephrin in human diabetic nephropathy," Kidney International, vol. 65, no. 6, pp. 2193-2200, 2004.

[98] M. Toyoda, D. Suzuki, T. Umezono et al., "Expression of human nephrin mRNA in diabetic nephropathy," Nephrology Dialysis Transplantation, vol. 19, no. 2, pp. 380-385, 2004.

[99] M. Cohen, S. Chen, F. Ziyadeh et al., "Evidence linking glycated albumin to altered glomerular nephrin and VEGF expression, proteinuria, and diabetic nephropathy," Kidney International, vol. 68, no. 4, pp. 1554-1561, 2005. 
[100] L. Chun-Liang, W. Feng-Sheng, H. Yen-Chen et al., "Modulation of Notch-1 signaling alleviates vascular endothelial growth factor-mediated diabetic nephropathy," Diabetes, vol. 59, no. 8, pp. 1915-1925, 2010.

[101] V. Garovic, S. Wagner, L. Petrovic et al., "Glomerular expression of nephrin and synaptopodin, but not podocin, is decreased in kidney sections from women with preeclampsia," Nephrology Dialysis Transplantation, vol. 22, no. 4, pp. 1136-1143, 2007.

[102] K. Koop, M. Eikmans, H. J. Baelde et al., "Expression of podocyte-associated molecules in acquired human kidney diseases," Journal of the American Society of Nephrology, vol. 14, no. 8, pp. 2063-2071, 2003.

[103] A. Fukuda, S. Fujimoto, S. Iwatsubo, H. Kawachi, and K. Kitamura, "Effects of mineralocorticoid and angiotensin II receptor blockers on proteinuria and glomerular podocyte protein expression in a model of minimal change nephrotic syndrome," Nephrology, vol. 15, no. 3, pp. 321-326, 2010.

[104] J. Mao, Y. Zhang, L. Du, Y. Dai, C. Yang, and L. Liang, "Expression profile of nephrin, podocin, and CD2AP in chinese children with MCNS and IgA nephropathy," Pediatric Nephrology, vol. 21, no. 11, pp. 1666-1675, 2006.

[105] A. T. Lahdenkari, M. Kestilä, C. Holmberg, O. Koskimies, and H. Jalanko, "Nephrin gene (NPHS1) in patients with minimal change nephrotic syndrome (MCNS)," Kidney International, vol. 65, no. 5, pp. 1856-1863, 2004.

[106] A. Shono, H. Tsukaguchi, A. Kitamura et al., "Predisposition to relapsing nephrotic syndrome by a nephrin mutation that interferes with assembly of functioning microdomains," Human Molecular Genetics, vol. 18, no. 16, pp. 2943-2956, 2009.

[107] P. N. Furness, L. L. Hall, J. A. Shaw, and J. H. Pringle, "Glomerular expression of nephrin is decreased in acquired human nephritic syndrome," Nephrology Dialysis Transplantation, vol. 14, pp. 1234-1237, 1999.

[108] W. Huh, D. J. Kim, M. K. Kim et al., "Expression of nephrin in acquired human glomerular disease," Nephrology Dialysis Transplantation, vol. 17, no. 3, pp. 478-484, 2002.

[109] T. Srivastava, J. M. Whiting, R. E. Garola et al., "Podocyte proteins in galloway-mowat syndrome," Pediatric Nephrology, vol. 16, no. 12, pp. 1022-1029, 2001.

[110] S. X. Wang, M. P. Rastaldi, A. Patari, H. Ahola, E. Heikkila, and H. Holthofer, "Patterns of nephrin and a new proteinuria-associated protein expression in human renal diseases," Kidney International, vol. 61, pp. 141-147, 2002.

[111] S. Doublier, V. Ruotsalainen, G. Salvidio et al., "Nephrin redistribution on podocytes is a potential mechanism for proteinuria in patients with primary acquired nephrotic syndrome," American Journal of Pathology, vol. 158, no. 5, pp. 1723-1731, 2001.

[112] J. Patrakka, V. Ruotsalainen, I. Ketola et al., "Expression of nephrin in pediatric kidney diseases," Journal of the American Society of Nephrology, vol. 12, no. 2, pp. 289-296, 2001.

[113] N. Guan, J. Ding, J. Zhang, and J. Yang, "Expression of nephrin, podocin, $\alpha$-actinin, and WT1 in children with nephritic syndrome," Pediatric Nephrology, vol. 18, pp. 11221127, 2003.

[114] S. R. Hingorani, L. S. Finn, J. Kowalewska, R. A. McDonald, and A. A. Eddy, "Expression of nephrin in acquired forms of nephritic syndrome in childhood," Pediatric Nephrology, vol. 19, pp. 300-305, 2004.

[115] A. Hara, T. Wada, K. Furuichi et al., "Blockade of VEGF accelerates proteinuria, via decrease in nephrin expression in rat crescentic glomerulonephritis," Kidney International, vol. 69, no. 11, pp. 1986-1995, 2006.

[116] T. Nakatsue, H. Koike, G. D. Han et al., "Nephrin and podocin dissociate at the onset of proteinuria in experimental membranous nephropathy," Kidney International, vol. 67, no. 6, pp. 2239-2253, 2005.

[117] H. Yuan, E. Takeuchi, G. A. Taylor, M. Mclaughlin, D. Brown, and D. J. Salant, "Nephrin dissociates from actin, and its expression is reduced in early experimental membranous nephropathy," Journal of the American Society of Nephrology, vol. 13, no. 4, pp. 946-956, 2002.

[118] D. R. Abrahamson, "Glomerulogenesis in the developing kidney," Seminars in Nephrology, vol. 11, no. 4, Article ID 1947493, pp. 375-389, 1991.

[119] M. Ohlson, J. Sörensson, K. Lindström, A. M. Blom, E. Fries, and B. Haraldsson, "Effects of filtration rate on the glomerular barrier and clearance of four differently shaped molecules," American Journal of Physiology, vol. 281, no. 1, pp. F103-F113, 2001.

[120] K. Sison, V. Eremina, H. Baelde et al., "Glomerular structure and function require paracrine, not autocrine, VEGFVEGFR-2 signaling," Journal of the American Society of Nephrology, vol. 21, no. 10, pp. 1691-1701, 2010.

[121] R. R. Foster, M. Saleem, P. Mathieson, D. Bates, and S. Harper, "Vascular endothelial growth factor and nephrin interact and reduce apoptosis in human podocytes," American Journal of Physiology, vol. 288, no. 1, pp. F48-F57, 2005.

[122] R. R. Foster, R. Hole, K. Anderson et al., "Functional evidence that vascular endothelial growth factor may act as an autocrine factor on human podocytes," American Journal of Physiology, vol. 284, no. 6, pp. F1263-F1273, 2003.

[123] D. Veron, K. J. Reidy, C. Bertuccio et al., "Overexpression of VEGF-A in podocytes of adult mice causes glomerular disease," Kidney International, vol. 77, no. 11, pp. 989-999, 2010.

[124] C. H. Ku, K. E. White, A. Dei Cas et al., "Inducible overexpression of sFlt-1 in podocytes ameliorates glomerulopathy in diabetic mice," Diabetes, vol. 57, no. 10, pp. 2824-2833, 2008.

[125] F. Guan, G. Villegas, J. Teichman, P. Mundel, and A. Tufro, "Autocrine VEGF-A system in podocytes regulates podocin and its interaction with CD2AP," American Journal of Physiology, vol. 291, no. 2, pp. F422-F428, 2006.

[126] J. Müller-Deile, K. Worthmann, M. Saleem, I. Tossidou, H. Haller, and M. Schiffer, "The balance of autocrine VEGFA and VEGF-C determines podocyte survival," American Journal of Physiology, vol. 297, no. 6, pp. F1656-F1667, 2009. 

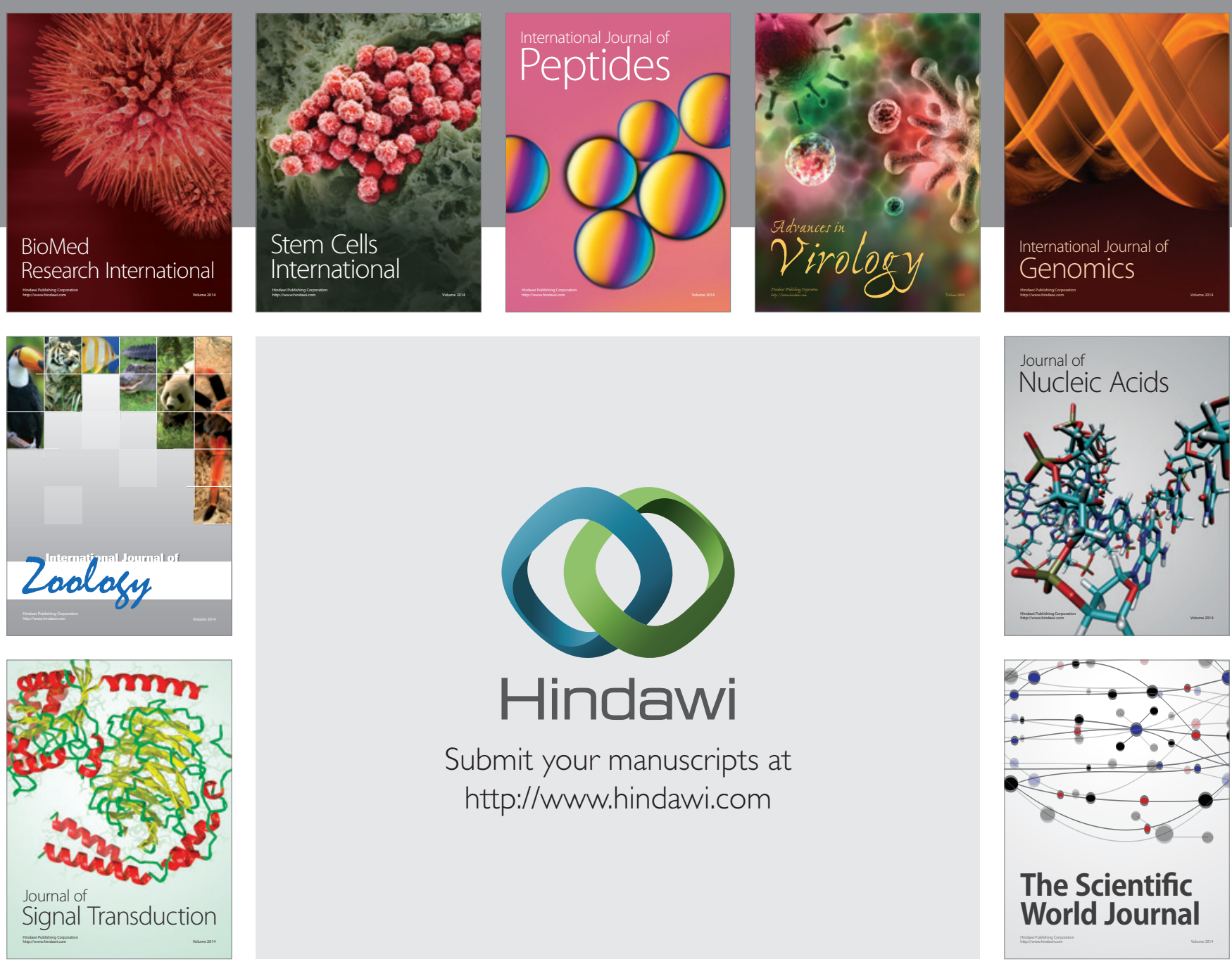

Submit your manuscripts at

http://www.hindawi.com
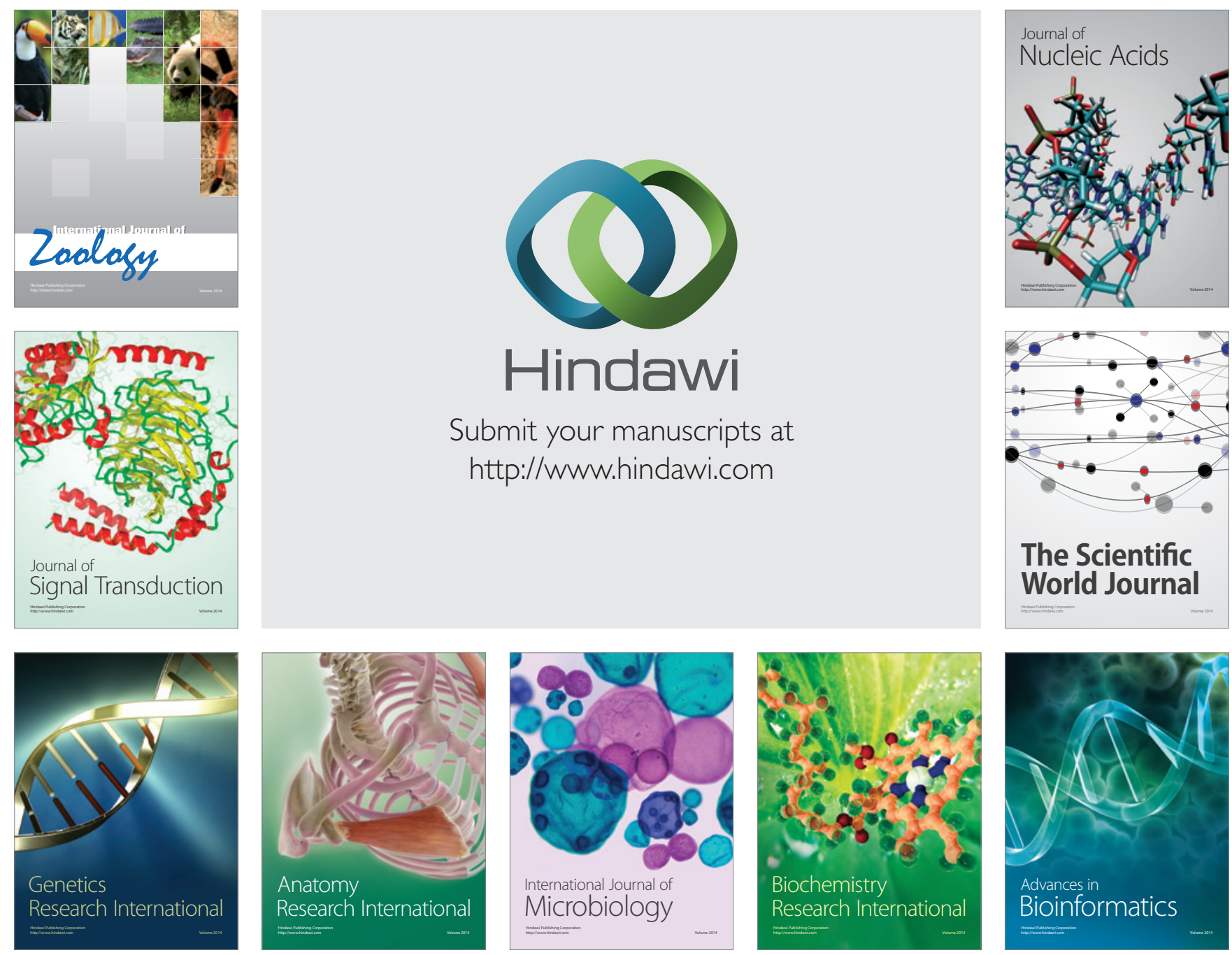

The Scientific World Journal
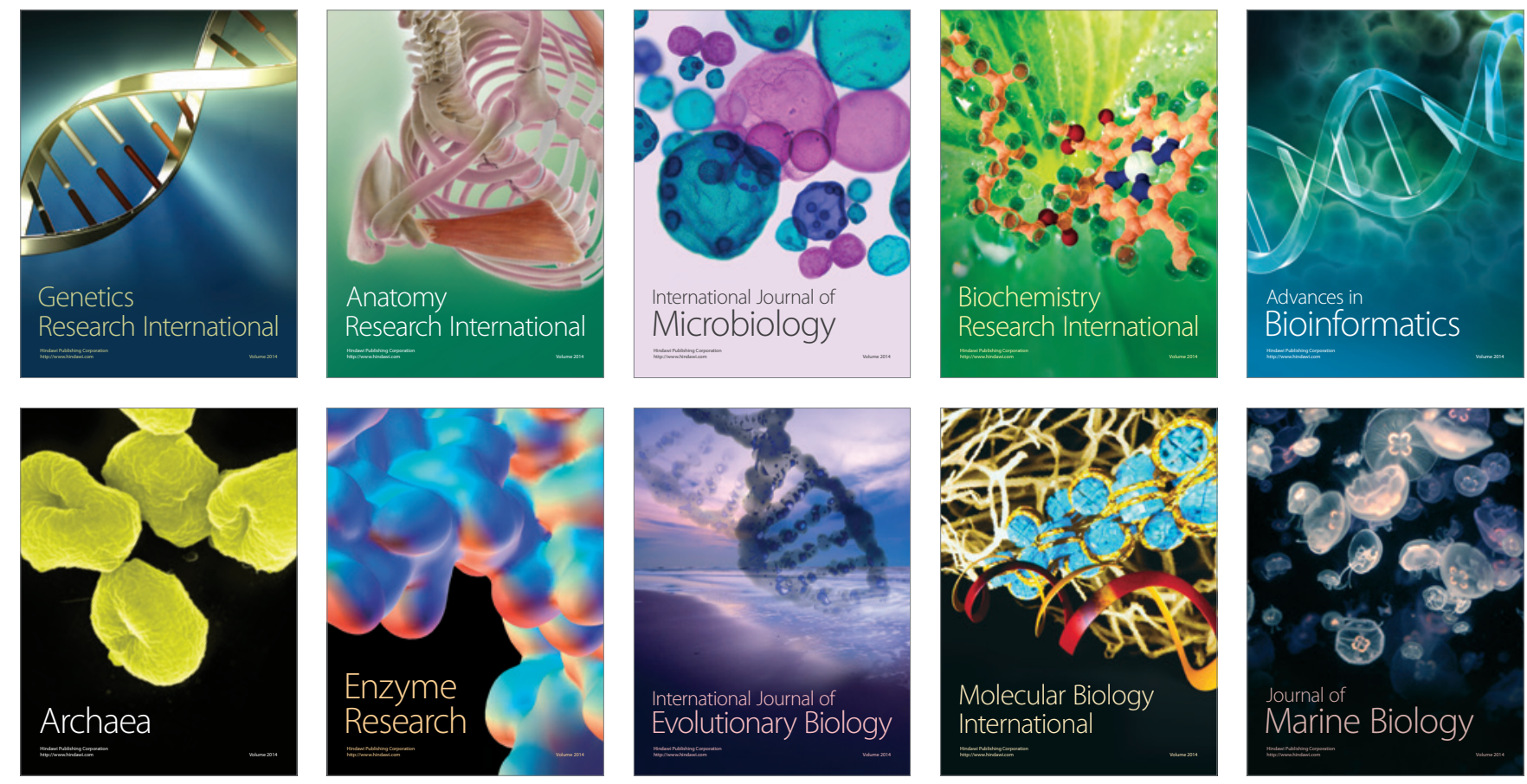